\title{
The instrument response function in air-based scanning tunneling microscopy
}

\author{
P. Fraundorf and J. Tentschert \\ Physics \& Astronomy, U. Missouri-StL (63121), \\ Corporate Research, Monsanto (63167), \\ Physics, Washington U. (63130), \\ St. Louis, MO, USA
}

(October 4, 2018)

\begin{abstract}
The distinction between point and line resolution in transmission electron microscopy (TEM) arises because an ability to image sub- $0.2 \mathrm{~nm}$ fringes is a necessary, but not a sufficient, condition for imaging individual atoms. In scanned tip microscopy, as in TEM, empirical data on instrument response should precede assertions about point resolution. In the "slow scan limit", time-domain noise and geometry effects decouple, and tip shape can take on the role of a 2-dimensional impulse response function. We indicate here that nuclear track pits can be used to quantitatively measure tip geometry with nanometer-scale resolution in three dimensions, that stationary tip images provide a robust measure of time-domain instabilities, and that when these data are taken before and after imaging an unknown, images with instrument response quantitatively constrained by experiment are possible. Specimen-induced tip effects also become measurable in situ. Also Ultramicroscopy $\mathbf{3 7}$ (1991) 125-129.
\end{abstract}

06.30.Bp, 07.79.-v, 61.16.Ch, 87.64.Dz

\section{INTRODUCTION}

In the literature of air-based scanned tip microscopy, images with $0.2 \mathrm{~nm}$ (or larger) fringes are often described as "atomically-resolved". $\Theta$ The assumption that fringe spacings provide an upper limit on point resolution in tunneling images was plausible, prior to the availability of recent eridence: (a) that tunneling in air or poor vacuum generally involves mechanical contact between specimen and tip (b) that "corrugation depths" between $0.2 \mathrm{~nm}$ rows in tunneling images of $\langle 001\rangle$ graphite and other layered structures can be many tenths of $\mathrm{nm}, \mathrm{B}$ (c) that the above images can become increasingly noise-free as tip sharpness decreases and mechanical contact between specimen and tip increases, and (d) that scanning force images unlikely to have point resolutions on the atomic scale also show atomic scale periodicities. ${ }^{-}$Mechanical contact between specimen and tip (always present in repulsive van der Waals force microscopy) leaves open the possibility that a periodic raft of specimen atoms, and not the tip itself, is sliding across the specimen, averaging periodicity information over distances much larger than a single unit cell. The ease of imaging fringes on layered structures thus provides evidence that lattice fringes alone, as in transmission electron microscopy, provide no proof of point resolution alone.

For this reason, and because the instrument response function in scanned probe microscopy introduces artifacts in the time domain as well as in space, researchers involved in air-based scanned tip studies should be at least as cautious as electron microscopist $\mathbf{3}$ in documenting their instrument's response. Because of the serial mode of data acquisition in scanning tunneling, spatial and temporal aspects of instrument response may require separate measurements. We show here how the elements for characterizing time domain instrument response are already present in the literature, and how nuclear particle track pits (etched 0 or unetched 7 ) provide a means for measuring the three-dimensional interaction profile for a given tip, while on site in the microscope.

\section{COMPONENTS OF INSTRUMENT RESPONSE}

Measuring instruments generally start with some property associated with a specimen, and end up producing a data object for analysis. For example, the contrast transfer function in high-resolution TEM begins with the electron wave-function at the exit surface of the specimen, and produces an image which may contain partial information on exit surface deBroglie phase from point to point. In scanned probe instruments, one begins with the profile from point to point of another quantity (e.g. the piezo height $z(x, y)$ associated with current $I$ at bias voltage $V$ ). From the resulting image we seek information on the local profile of some height $h$ (i.e., of constant current for an arbitrarily sharp tip at constant conductivity $I / V)$. We refer here to instrument response as that function $R$ which maps the local profile $h\left(x^{\prime}, y^{\prime}\right)$ into the collected image $z(x, y)$, i.e., it is the function for which $z(x, y)=R\left(x, y,\left\{h\left(x^{\prime}, y^{\prime}\right) \forall x^{\prime}, y^{\prime}\right\}\right)$. 
Because the $z(x, y)$ values are obtained sequentially in time, the geometric aspects of $R$ are intertwined with timedomain components of instrument response which are at best partially known. However, in the slow scan limit, for which the tip is allowed to assume a steady state with each new value of $x$ and $y$ (i.e., when the feedback loop is fast enough to follow specimen topography for the scan rates in use), the geometric and time domain effects can be separated, and $z(x, y)$ can be written as:

$$
z(x, y)=R_{g}\left(x, y,\left\{h\left(x^{\prime}, y^{\prime}\right) \forall x^{\prime}, y^{\prime}\right\}\right)+N(x, y),
$$

where $R_{g}$ is a strictly geometric version of the instrument response, and $N(x, y)$ respresents the time-domain noise in the system. The next section discusses procedures for measuring the second moment statistics of $N(x, y)$, as well as Bayesian (i.e., best guess) strategies for subtracting $N(x, y)$ from images therewith. The remaining function $R_{g}$ depends only on the details of tip structure, and of course on the signal being measured. In the simplest case of a profile which is: (i) dominated by tunneling current through the infinitesimal point on the tip nearest the specimen, and (ii) dependent on gap distance but not on local curvature, composition, etc., $R_{g}$ takes the form $\mathrm{g}$ of

$$
R_{g}(x, y)=h(0,0)+\max \left[\left\{h\left(x+x^{\prime}, y+y^{\prime}\right)-t\left(x^{\prime}, y^{\prime}\right)\right\} \forall x^{\prime}, y^{\prime}\right] .
$$

Here $t\left(x^{\prime}, y^{\prime}\right)$ are $z$-coordinates of the tip surface while it is pointing toward a specimen in the negative $z$-direction, with the tip positioned horizontally so that its scan coordinates are $\{x, y\}=\{0,0\}$, and vertically so that $t(0,0)$ equals the height of the specimen at the scan origin. In practice, of course, we ignore absolute heights and consider relative heights only. Note here that $t\left(x^{\prime}, y^{\prime}\right)$ serves as a kind of impulse response function, since it predicts the shape of the noise-subtracted image for the limiting case of an infinitely sharp specimen. In low-magnification air-based scanning tunneling microscopy on rough specimens, where tip geometry has major effects and the nature of tip-specimen interaction is poorly known, measured profiles of the effective tip-interaction geometry $t_{e f f}\left(x^{\prime}, y^{\prime}\right)$ provide useful estimates of the impulse response in these images as well. A simple method for measuring profiles of tip-interaction geometry in the microscope, on the nanometer scale, is therefore discussed in the section on tip fingerprints below.

\section{STATIONARY TIP IMAGES}

Before discussing use of stationary tip images to determine the power spectrum of $\mathrm{N}(\mathrm{x}, \mathrm{y})$, we should first discuss violations of the slow scan assumption. Time-domain artifacts associated with response of the feedback loop to slope changes on the specimen include spikes, associated with rapid encounters by the tip with slopes that rise too quickly, and "hand-glide" artifacts associated with slopes which drop away too soon. Imaging of objects more than once, with different scan rates and amplitudes, can help detect features like these, and comcomitant violation of the assumptions which go into eq. (11). Slowing the scan rate, of course, entails compromise itself, not the least of which in air is the amount of thermal drift that takes place during image acquisition.

The statistical properties of $N(x, y)$ in eq. (1), given its appropriateness for a specific set of images, have been examined, and statistical removal by one-dimensional 1 and two-dimensional 10 Wiener optimal filtering has been proposed provided a suitable model for the noise power spectrum is available. These filtering techniques are a special case of Bayesian background subtraction, a techniqu which is now finding application in place of Fourier averaging for the removal of noise from HREM images as well.11 For the empirical background model in STM, Stoll and Marti12 have proposed using stationary tip images (defined here as images taken with the $x$ and $y$ tip scan-range set to zero). These stationary tip images are of special importance because, like HREM images of aperiodic material used in quantifying instrument contrast transfer, they can be obtained: (i) on the same specimen, (ii) under the same imaging conditions, and (iii) at almost the same time as the image whose instrument response is being considered.

We note in passing that stationary tip images, also like HREM images of aperiodic material, provide a robust measure of the effect of time-domain instability. They are quite sencitive to changes in instrument and environment, but insensitive to changes in tip and often feedback loop settings. 13 Hence they can be used to measure instrument performance as well as the effects of site instability on that performance.

\section{FINGERPRINTS OF THE TIP}

After removal of time-domain artifacts with help from stationary tip and multiple-scan images, the task of relating $z(x, y)$ to some local property of the specimen remains. In HREM, the analogous setp involves determining the contrast transfer function for a given image, with help from aperiodic structure in the image as well as prior information on instrument response. A specimen-independent fingerprint of instrument response in scanned probe images does not 
appear to be available. The empirical approach is therefore simple: constrain the tip response before and after taking an unknown image by observations on a known.

The simplest known for this purpose would be an otherwise flat surface populated with identical Poisson-distributed delta-functions (i.e., atomically sharp peaks). In principle such specimens can exist for both tunneling and force microscopy, but in practice they remain to be identified. However, the nuclear particle track formation mechanism can already provide geometrically uniform, nanometer-sized damage regions capable of systematic enlargement by etching to diameters well over a micron.

Fig. 1 shows an image obtained by averaging scanning tunneling images of four nearby $50 \mathrm{~nm}$ diameter etched and $\mathrm{Au}-\mathrm{Pd}$ coated nuclear particle track pits 14 Individual data points in this pit profile showed standard deviations of only $2 \mathrm{~nm}$, and the standard deviation image indicated that spatial correlations in these errors, if any, were confined to regions of steep slope. The contours in Fig. 1 represent levels of three standard deviations in the average rms pixel spread from image to image. A well defined and repeatable structure of ledges is present. Resolution is near to (or better than) $5 \mathrm{~nm}$ in all directions, and we suspect this is limited in part by grain size in the Au-Pd coating, and in part by variations (obviously quite small) from pit to pit.

This geometric pit profile of Fig. 1 can be inverse-transformed (see inset) to provide a three-dimensional view of those portions of the tip-interaction profile which we can geometrically (and/or electronically) bring into contact with the inside of a $50 \mathrm{~nm}$ diameter ring. 15 In other words, quantified pit images like those shown in Fig. 1 provide "better-than-convex envelope' characterizations of $t_{e f f}\left(x^{\prime}, y^{\prime}\right)$, in this case with quantitative spatial resolution in three dimensions on the order of one-tenth of the hole diameter. These pit images can be obtained before and after imaging an unknown specimen, thus allowing one to characterize tip modifications as well as tip structure.10 Use of smaller and larger pits allows characterization of tip geometry on smaller and larger size scales as well. We expect the strategy will allow formation of reproducible pits in the nm size range for scanning force work, although as pit size decreases for scanning tunneling work the problem of texture in the conductive coating is expected to increase in severity.

\section{CONCLUSIONS}

It is, of course, possible to compare unknown specimen images with other specimen images, and with model expectations based on prior information, and thereby to do a great deal of microscopy without hard information on instrument response. In vacuum-based scanned probe microscopy, prior information about both specimen and tip may be adequate to obviate the need for empirical data on instrument response. In air- and liquid- based scanned probe study, however, a strength of the technique is that the instruments are capable of examining true unknowns. But before structure images, in the electron microscopic sense 3 , can be obtained in general, an empirical protocol for inferring (and removing) the effects of instrument response is necessary.

We have illustrated how theoretically, in the slow-scan limit, the time-domain and geometric components of scanned probe instrument response can be decoupled, and how the geometric interaction profile of the scanned tip in that case can become a two-dimensional impulse response function. Further, we have shown how stationary tip images may serve as a robust source of information on time-domain instabilities, obtainable while the tip is in place on the unknown of interest. Finally, we illustrated that the geometric profile of a tip in air (or liquid or vacuum) can be measured with $5 \mathrm{~nm}$ or better resolution, in the microscope before and after examination of an unknown specimen. This confirms our earlier observation that nuclear particle tracks are a promising tool in this regard, and further opens the door to quantitative studies of the effect of specimens on tips: a needed complement when tip-induced changes to the specimen are being considered. Overall, feeling the need because we have been "spoiled" by elegant tools for characterizing instrument response in electron phase contrast imaging, we: (i) have proposed a strategy for obtaining data on scanned probe instrument response almost on an image-by-image basis, and (ii) where we have not seen the components used elsewhere have illustrated their workability.

\footnotetext{
${ }^{1}$ e.g. R. J. Colton, S. M. Baker, J. D. Baldeschwieler, and W. J. Kaiser, Appl. Phys. Lett. 51 (1987) 305; T. R. Albrecht and C. F. Quate, J. Appl. Phys. 62 (1987) 2599; Y. Nakagawa, A. Ishitani, T. Takahagi, H. Kuroda, H. Tokumoto, M. Ono and K. Kajimura, J. Vac. Sci. Technol. A8 (1990) 262.

${ }^{2}$ M. Kuwabara, W. Lo and J. C. H. Spence, J. Vac. Sci. Technol. A7 (1989) 2745.

${ }^{3}$ cf. J. Schneir, R. Sonnenfeld, P. K. Hansma and J. Tersoff, Phys. Rev. B34 (1986) 4979.

${ }^{4}$ e.g. R. G. Miller and P. J. Bryant, J. Vac. Sci. Technol. A7 (1989) 2879.
} 
${ }^{5}$ cf. J. Spence, Experimental High-Resolution Electron Microscopy (Oxford Univ. Press, New York, 1981 and 1988), 1st and 2nd ed.

${ }^{6}$ cf. R. L. Fleischer, P. B. Price and R. M. Walker, Nuclear Tracks in Solids (U. California Press, 1975).

${ }^{7}$ P. Fraundorf and J. Tentschert, Proc. Intern. Congress on Electron Microscopy 1 (1990) 584.

${ }^{8}$ e.g. R. Möller, A. Esslinger and M. Rauscher, J. Vac. Sci. and Technol. A8 (1990) 434.

${ }^{9}$ S. I. Park and C. F. Quate, J. Appl. Phys. 62 (1987) 312.

${ }^{10}$ E. Stoll and A. Baratoff, Ultramicroscopy 25 (1988) 144.

${ }^{11}$ P. Fraundorf, Phys. Rev. Lett. 64 (1990) 1031, cond-mat/9711309, P. Fraundorf and K. Pollack, Ultramicroscopy 37 (1991) 72 .

12 E. Stoll and O. Marti, Surface Science 181 (1987) 229.

${ }^{13}$ L. Fei and P. Fraundorf, Abstracts to the 38th Midwest Solid State Conference (University of Nebraska in Lincoln, 1990), E2-3.

${ }^{14}$ J. Tentschert and P. Fraundorf, ibid., E2-2.

${ }^{15}$ P. Fraundorf, unpublished (1990).

${ }^{16}$ J. Tentschert and P. Fraundorf, Abstracts to the American Physical Society March 1991 Meeting (1990) submitted.

FIG. 1. Image obtained by averaging $32 \times 32$ pixel images of four $50 \mathrm{~nm}$ diameter etched, and Au-Pd coated, nuclear particle track pits in polycarbonate. The pit images were cut from larger 12-bit images obtained with an $8 \mu \mathrm{m}$ Digital Instruments head using a single mechanically formed Pt-Ir tip. The bottom of the pit is white, while the surrounding flat polycarbonate is dark. Contours (at intervals of 3 standard deviations in the rms pixel variation from image to image) are white in regions surrounding the pit, and dark in the deeper regions of the structure. The pit base contour (white) is round and approximately $50 \mathrm{~nm}$ in diameter, although it appears oval here because of different magnifications in the x and y scan directions. Depth from base to bottom is of the order of $20 \mathrm{~nm}$ (around 487 greyvalue units), based on rough z-gain calibrations using $30 \mathrm{~nm}$ collodial gold on graphite. The inset shows the resulting envelope (z-height exaggerated) for the $20 \mathrm{~nm}$ end of the tip used to take this data (see text). 
This figure "irfstmf1.gif" is available in "gif" format from: http://arxiv.org/ps/physics/9712003v1 